\title{
Percepción de desorden, riesgo y seguridad: la influencia del método Marcela Acuña-Rivera ${ }^{1}$, David Uzzell ${ }^{1}$ and Jennifer Brown ${ }^{2}$ \\ ${ }^{1}$ University of Surrey; ${ }^{2}$ London School of Economics
}

\begin{abstract}
Resumen
De acuerdo con varios estudios, la evaluación de incivilidades físicas y sociales de los lugares forma parte del proceso mediante el cual la gente estima su nivel de seguridad. El estudio que aquí se presenta investiga si tal supuesto se mantiene cuando a la gente se le permite expresar lo que piensa de un lugar antes de evaluar el nivel de desorden físico y social del mismo. En primer lugar, los participantes debían escribir sus impresiones sobre tres vecindarios con distintos niveles de desorden, y después evaluar mediante un cuestionario que tan desordenados e inseguros les parecían. El análisis cualitativo mostró que aun cuando los participantes mencionaron el desorden físico del lugar, sólo algunos de ellos hicieron referencia a cuestiones de crimen e inseguridad. El análisis cuantitativo reveló que mientras mas desordenado se evaluaba un lugar mayor inseguridad se percibía. Las conclusiones sugieren que, por un lado, la percepción de desorden de un lugar no siempre evoca respuestas de inseguridad y, por el otro, que la relación encontrada entre desorden e inseguridad puede deberse al método de investigación utilizado.
\end{abstract}

Palabras clave: Desorden, miedo al crimen, seguridad, percepción de riesgo, ambiente físico, ambientes residenciales.

\section{Perceptions of disorder, risk and safety: The method and framing effects}

\begin{abstract}
Several research studies have argued that people evaluate incivilities of places as part of the process of estimating how safe they might be. The study presented here examined whether such an assumption is upheld when people are allowed to express their thoughts about places before rating how disordered a place seems to them. British students evaluated three residential areas with different levels of disorder. First, participants had to write their impressions about the places and then rate how disordered, risky and unsafe the places seemed to them. The qualitative analysis showed that despite participants referred to physical disorder, only few participants mentioned crime and safety. Results from the quantitative analysis revealed that as the more disordered a place was rated the more unsafe it was considered. Findings suggest both that disordered places not always elicit unsafe concerns and that the so predicted relationship between disorder and safety maybe method dependant.
\end{abstract}

Keywords: Disorder, fear of crime, safety, risk perception, physical environment, residential environments. 


\section{Introducción}

La publicación de la "Teoría de las Ventanas Rotas" (o Broken Windows por su denominación en inglés) y sus implicaciones (Wilson, 1975; Wilson y Kelling, 1982) atrajeron el interés de políticos, diseñadores e investigadores de diversas disciplinas. Los partidarios de esta teoría argumentaban que las personas que perciben más desordenado un vecindario son las que se mostrarán más preocupadas por su seguridad; es decir, cuanta más incivilidad perciba, menos segura se sentirá una persona (Jackson, 2004a; Taylor, 2001a, 2001b; Wilson y Kelling, 1982). Por lo general, las incivilidades físicas se relacionan con señales que muestran que un lugar no se mantiene o se usa correctamente; las incivilidades sociales se asocian con un comportamiento desordenado e impredecible, problemático y amenazante. Según esta teoría, ambos tipos de incivilidades informan tanto a residentes como a extraños que el vecindario no está bajo control sino que, más bien, es un lugar desordenado.

Los lugares desordenados provocan miedo y ansiedad porque muestran indicios de vulnerabilidad e invitan a los infractores a entrar; como consecuencia, el deterioro, la desconfianza y el retraimiento se incrementan. (Brantingham y Brantingham, 1995; Brown, Perkins, y Brown, 2004; Doran y Lees, 2005; Farrall, Gray sid5314734y Jackson, 2007; Friedrichs y Blasius, 2003; Micel, Roccato y Rosato, 2004; Semmens, 2004). Por el contrario, los lugares ordenados ofrecen seguridad y tranquilidad porque muestran indicios de control social y vigilancia, y porque la gente que vive en ellos es percibida como cuidadosa y digna de confianza (Brown et al., 2004; Skogan, 1990; Taylor, 1987; Wilson y Kelling, 1982). 
Gran parte de la investigación sobre incivilidades en ambientes residenciales se ha centrado en la investigación de ciertas características físicas y sociales que, se dice, debilitan el control social informal y la estabilidad del vecindario, e incrementan la preocupación por la seguridad tanto personal como de los inmuebles. Por ejemplo, se ha encontrado que la iluminación insuficiente, la novedad y la alta densidad física hacen que la gente se sienta menos segura (Painter, 1996). Asimismo se ha encontrado que la cantidad y el mantenimiento de la vegetación ejercen un efecto sobre el crimen, el miedo y la seguridad percibida (Kuo, Bacaioca y Sullivan, 1998; Kuo y Sullivan, 2001). La estabilidad del vecindario, la apropiación del espacio por parte de los residentes, un fuerte sentido de comunidad y la confianza social provocan sentimientos de bienestar y seguridad (Brunson, Kuo y Sullivan, 2001; Garcia, Taylor y Brian, 2007; Markowitz, Bellair, Liska y Liu, 2001; Ross, Reynolds y Geis, 2000; Schwitzer, Kin y Mackin, 1999). Sin embargo, estas conclusiones deben investigarse más a fondo ya que algunos resultados parecen contradictorios, y otros investigadores han encontrado que existen otros factores que median o moderan el efecto que las incivilidades tienen sobre la percepción de seguridad, tales como la estructura de la comunidad, el apego al lugar y la confianza social (Brown y Perkins, 2001; Franzini, O’Brien, Murray y O’Campo, 2008; Friedrichs y Blasius, 2003; Skogan, 1990; Taylor, 1996; Van Beek Gert, 2005; Walkalate, 1998).

El valor de la "Teoría de las Ventanas Rotas (TVR)" es que Wilson y Kelling cambiaron del enfoque tradicional, que estudiaba las diferencias individuales como causas del crimen, a una aproximación más de tipo ambiental, la cual subrayaba la relevancia de las condiciones físicas y sociales del lugar como factores que pueden explicar la percepción de seguridad y el miedo a la victimización. Sin embargo, aun cuando 
reconocieron que el nivel de desorden varía de vecindario a vecindario, no explicaron los procesos subyacentes que podrían explicar tales variaciones.

La TVR y los posteriores planteamientos conceptuales, han recibido críticas por su determinismo ambiental y por no incorporar factores psicosociales que puedan explicar la percepción de desorden e inseguridad. Actualmente, existe una creciente preocupación por las deficiencias conceptuales y metodológicas que este enfoque ha demostrado a lo largo de los años (Farrall, Banni ster, Ditton y Gilchrist, 1997; Ferraro y LaGrange, 1987; Hale, 1996; Jackson, 2004b; Semmens, 2004). Por ejemplo, a nivel conceptual se le ha criticado por no tener en cuenta la opinión y los distintos puntos de vista de los participantes. Algunos investigadores también argumentan que el uso generalizado de encuestas ha descontextualizado el problema y no se ha retratado adecuadamente la variedad de significados y emociones implicados. Además gran parte de la investigación en este campo hace muy poca distinción, tanto conceptual como operacional, entre términos tales como miedo, preocupación, seguridad, riesgo, crimen y victimización (Ferraro y LaGrange, 1987; Jackson, 2004b).

Las preocupaciones metodológicas giran en torno a la forma en que se operacionalizan conceptos clave como el miedo. La investigación en el campo no distingue entre tipos de delitos ni se define su contexto espacio-temporal y social; hecho que ha limitado tanto su desarrollo conceptual como la calidad de los datos recolectados (Farral et al., 1997). Por ejemplo, a menudo se pregunta a los participantes sobre el 'crimen' en general o 'cuán seguros/temerosos/preocupados/inquietos se sienten al caminar solos en una zona determinada. Ciertamente, es necesario hacer distinciones conceptuales y 
operaticionales entre los términos empleados y las situaciones planteadas, y establecer además el contexto en el que ocurren tales situaciones.

Otras prácticas metodológicas, como el uso excesivo de encuestas y la formulación de las preguntas empleadas, acentúan aun más la deficiencia del campo. Farrall y sus colaboradores realizaron un estudio para comparar los resultados que se obtienen a partir de preguntas abiertas o cerradas, y encontraron que los mismos participantes daban respuestas distintas a preguntas muy similares o idénticas, y que ello dependía del tipo de pregunta formulada. Fattah (1993) coincide con el punto de vista de Farrall, y argumenta que el hecho de que se obtengan resultados distintos según se empleen preguntas abiertas o cerradas, se debe a que estas últimas dirigen las respuestas de los participantes. Así, el uso de métodos cuantitativos y cualitativos puede originar conclusiones diferentes, atribuibles más al método empleado que a un cambio en la respuesta de los participantes.

Considerando las deficiencias descritas anteriormente, el estudio que aquí se presenta investiga si la premisa de que "los lugares desordenados física y socialmente evocan sentimientos de inseguridad y miedo a la victimización”, se comprueba cuando se le permite a la gente expresar lo que en realidad piensa acerca de los lugares. Si, cuando se pregunta a la gente acerca de tres áreas residenciales con distintos niveles de desorden, manifiestan preocupación por el peligro y la seguridad, o subrayan otros atributos observados e inferidos acerca de esos lugares; y si, en sus evaluaciones del nivel de desorden, riesgo y seguridad de las mismas áreas residenciales, se produce un efecto del desorden en las inferencias de seguridad y victimización. 


\section{Método}

\section{Participantes}

Se empleó una muestra de 120 estudiantes británicos para el análisis de este estudio. El $56.7 \%$ eran mujeres y el $43.3 \%$ hombres; el $78.4 \%$ tenía entre 18 y 25 años (78.4\%) y en su mayoría eran estudiantes de grado (61.7\%). Para contactar a los participantes, se envió un correo electrónico a distintas facultades de una universidad inglesa, pidiéndoles su participación voluntaria.

\section{Los tres lugares evaluados}

Para elaborar los materiales a evaluar, se tomó una fotografía panorámica diurna de un vecindario en una zona residencial altamente marginal, sin gente (Figura 1: El lugar tal como es). Con base en las premisas conceptuales y contribuciones empíricas de la Teoría de las Ventanas Rotas y de las perspectivas sobre incivilidades y desorden,, se crearon dos variaciones del Lugar tal como esl. En primer lugar, se identificaron las incivilidades físicas que se dice caracterizan a los vecindarios desordenados. Entre ellas se encuentran: mantenimiento global, ventanas rotas, basura, edificios con puertas y ventanas entabladas, alta densidad física, iluminación insuficiente, farolas rotas, grafiti, y propiedades y vehículos vandalizados y abandonados (Hale, 1996; Jackson, 2004a; Skogan, 1990; Taylor, 1987, 2001a, 2001b; Van Beek Gert, 2005; Wilson y Kelling, 1982). 
Figura 1. El Lugar Tal Cual

Esta fotografía muestra el lugar como es en realidad.

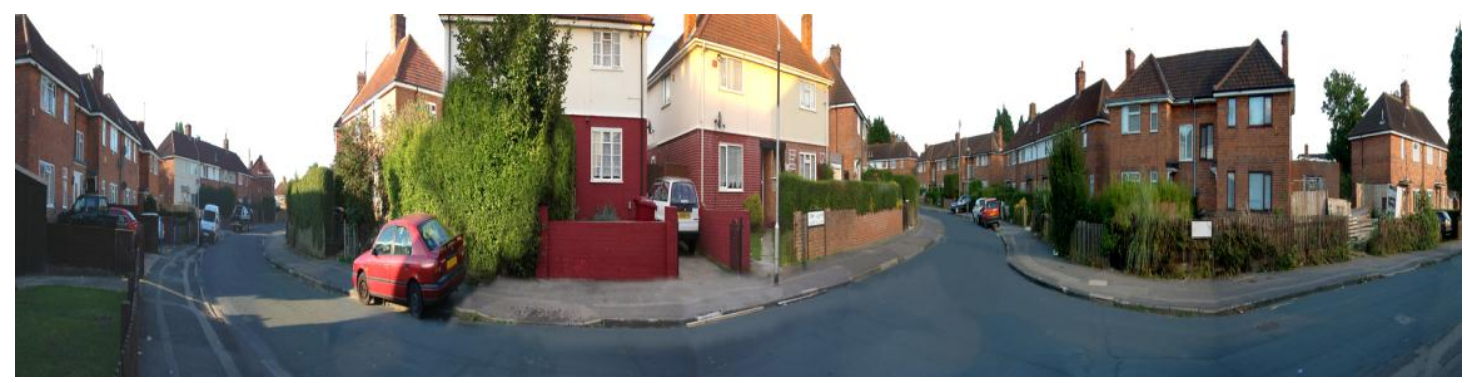

En segundo lugar, empleando una aplicación informática de diseño, se manipularon las características físicas como la limpieza de calles y viviendas, las condiciones de paredes y ventanas, así como las condiciones y número de autos y vegetación, con el fin de crear dos lugares adicionales: 1) Un lugar más deteriorado (Figura 2: Lugar deteriorado) con señales de incivilidades y deterioro, más autos, suciedad, vegetación sin podar, ventanas rotas y grafiti; y, 2) Un lugar mejorado (Figura 3: Lugar mejorado), el cual luce mejor cuidado, más prolijo y limpio, con menos autos, ventanas más nuevas y con más vegetación y mejor podada. El propósito de estas modificaciones era explorar en qué medida la percepción del desorden, el riesgo y la seguridad cambian en función de las condiciones físicas de los lugares. 
Figura 2. El Lugar Deteriorado

El lugar real se modificó para crear un lugar más deteriorado y menos atractivo. La idea general era crear un lugar descuidado que evidenciara signos de deterioro y abandono.

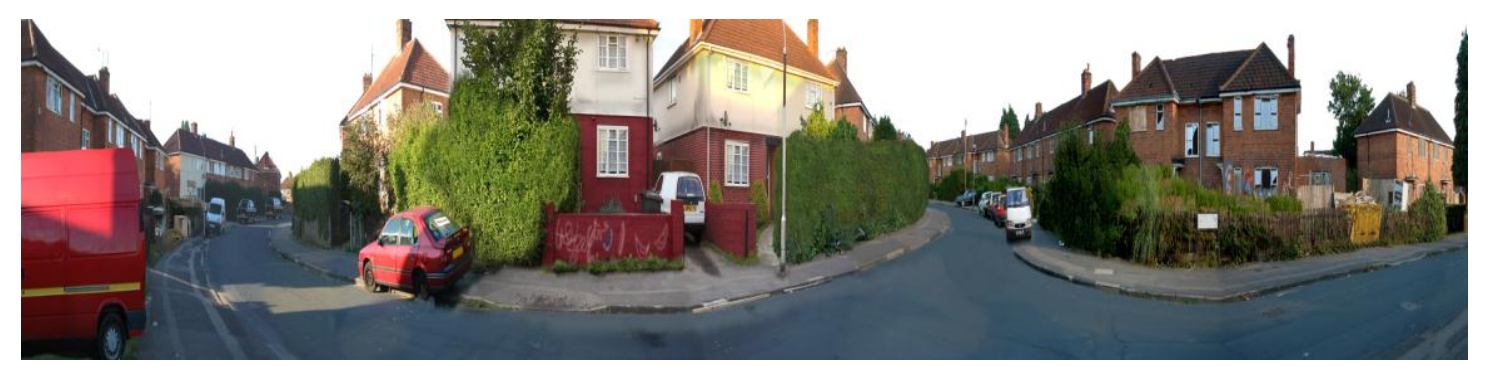

Figura 3. El Lugar Mejorado

El lugar real se manipuló para crear un lugar embellecido y más atractivo. Se añadió vegetación cuidada y podada con el fin de crear un lugar más verde, privado y cuidado.

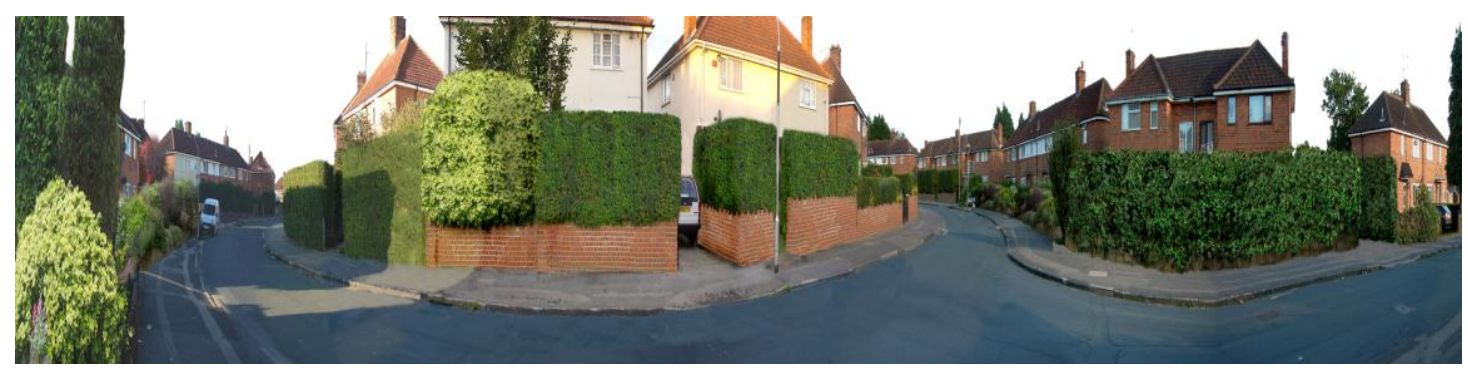

\section{Instrumentos y variables}

Se elaboró un cuestionario para evaluar la percepción de desorden físico y social, la percepción de riesgo, y de seguridad. El cuestionario contenía dos partes principales. La Parte I planteaba una pregunta abierta que pedía a los participantes que escribieran al menos cinco palabras o frases que expresaran adecuadamente lo que pensaban cuando veían los lugares por primera vez. En la Parte II del cuestionario se presentaba a los participantes una serie de afirmaciones sobre el desorden físico y social, el riesgo y la seguridad. Las variables se evaluaron como sigue: 
Desorden. El desorden físico y social se evaluó mediante una pregunta abierta y 40 preguntas cerradas relacionadas con el mantenimiento de la zona residencial, incivilidades físicas y sociales, legibilidad, deterioro, y sentido de la comunidad. Mediante una escala de 1 a 10, en la que 1 representa "para nada" y 10 "mucho", los participantes debían evaluar el grado en el que cada pregunta describía el lugar mostrado en la fotografía.

La percepción de riesgo se midió mediante 21 preguntas que evaluaban la vulnerabilidad percibida (o la susceptibilidad percibida de enfrentar situaciones o eventos amenazantes que pudieran causarles daño a ellos o a las cosas que valoran),el control (o la estimación de la capacidad personal para evitar o mitigar riesgos y sus consecuencias), y la frecuencia de ocurrencia de ciertos peligros (eventos, situaciones o personas) que pudieran causarles daño. Mediante una escala de tipo Likert, en la que 1 significaba "para nada" y 7 "mucho", los participantes debían evaluar que tan probable era que se sintieran vulnerables o bajo control en relación con ciertos delitos al ver las fotografías.

La seguridad percibida se midió mediante cuatro preguntas cerradas. Los participantes debían evaluar en una escala tipo Likert similar a la anterior, cuán inseguros o intranquilos podían sentirse en los lugares evaluados.

\section{Procedimiento}

Los participantes debían evaluar las fotografías una a una. El investigador entregaba la primer fotografía al participante y le pedía que la mirara bien y completara un 
cuestionario. Cuando terminaba, el investigador recogía la fotografía y el cuestionario y entregaba al participante la segunda foto y un nuevo cuestionario. Lo mismo se hizo para la tercera fotografia. Aun cuando los participantes evaluaron los tres lugares, el orden de presentación de las mismas se modificó para evitar que influyese en los resultados.

\section{Análisis y resultados}

¿Qué pensaron los participantes sobre el lugar en la fotografía?

Antes de responder las preguntas cerradas del cuestionario, se le pidió a los participantes que escribieran cinco palabras o frases que reflejaran su impresión de los lugares evaluados cuando los vieron por primera vez. Las respuestas a esta pregunta se codificaron y analizaron utilizando el método de análisis de contenido (Weber, 1990). Una vez identificadas las categorías y subcategorías que englobaban las respuestas de todos los participantes, se procedió a calcular la confiabilidad inter-jueces. Para ello, un investigador independiente codificó las respuestas de diez participantes y, mediante el uso del coeficiente Kappa de Cohen, se obtuvo un coeficiente aceptable de $r=.83$ (Miles y Huberman, 1994). Los resultados presentados resaltan los principales atributos percibidos por la mayoría de los participantes. Cabe mencionar que no todos los participantes se refirieron a todas las categorías y subcategorías descritas (Tabla I).

Cuatro categorías interrelacionadas surgieron del análisis. La primera categoría, características relacionadas con el lugar, recoge los atributos que se observaron o en ocasiones se infirieron del lugar en sí, como incivilidades físicas, densidad y privacidad. La segunda categoría, atribuciones sociales, incluye temas que se infirieron sobre el lugar pero que tienen un contenido social como nivel económico y amabilidad de la 
gente que vive en estas zonas. La tercera categoría, reacción personal, se refiere a la medida en que el lugar gustó a la gente, cómo se sintieron al verlo y cuán familiar les resultaba. La cuarta categoría, seguridad percibida, refleja la posibilidad de encontrarse en peligro.

Tabla I.

Categorías y subcategorías emergentes del análisis de los resultados mediante AC

\begin{tabular}{|c|l|c|l|}
\hline Categorías & \multicolumn{1}{|c|}{ Sub-categorías } & Categorías & \multicolumn{1}{|c|}{ Sub-categorías } \\
\hline & $\begin{array}{l}\text { Incivilidades físicas } \\
\text { Mantenimiento } \\
\text { RELACIONADOS } \\
\text { CON EL LUGAR } \\
\text { Privacidad física } \\
\text { Tipo de inmuebles } \\
\text { Densidad percibida } \\
\text { Tipo de área }\end{array}$ & $\begin{array}{c}\text { ATRIBde gente } \\
\text { Amabilidad } \\
\text { SOCIALES }\end{array}$ & \\
\hline $\begin{array}{l}\text { REACCIÓN } \\
\text { PERSONAL }\end{array}$ & $\begin{array}{l}\text { Atractivo } \\
\text { Reacción emocional } \\
\text { Familiaridad }\end{array}$ & $\begin{array}{l}\text { SEGURIDAD } \\
\text { PERCIBIDA }\end{array}$ & $\begin{array}{l}\text { Seguridad percibida } \\
\text { Peligro percibido } \\
\text { Incivilidades } \\
\text { sociales }\end{array}$ \\
\hline
\end{tabular}

Nota: Puede contarse a algunos participantes en más de una sub-categoría.

El Lugar Deteriorado evocó respuestas asociadas a su mantenimiento global y a incivilidades físicas y sociales, así como a su nivel económico y grado de peligrosidad. Es decir, el $73 \%$ de la muestra percibió incivilidades físicas en este lugar, el $45 \%$ lo percibió como descuidado (45\%) y el $17 \%$ como barrio de gente pobre. Alrededor del $35 \%$ de los participantes se refirió a este lugar como peligroso e inseguro, y en donde el crimen y el vandalismo son habituales. El Lugar tal como es evocó respuestas asociadas a su mantenimiento global e incivilidades físicas, su nivel económico y su atractivo. Este lugar se percibió principalmente como un vecindario tranquilo pero descuidado y desprolijo, con un nivel económico moderado; solo una pequeña parte (12\%) pensó que era un lugar peligroso. El Lugar Mejorado evocó respuestas asociadas a su mantenimiento global y tranquilidad, su nivel de privacidad, vegetación, atractivo y 
seguridad percibida. Se considero un lugar cuidado, tranquilo y verde. Alrededor del $30 \%$ de la muestra se refirió a este como un lugar seguro y atractivo.

En general, los resultados anteriormente descritos sugieren que aunque el $70 \%$ de los participantes percibió El Lugar Deteriorado como el lugar más deteriorado, desprolijo, vandalizado y descuidado, y el $42 \%$ infirió la presencia de incivilidades sociales, solo el $33 \%$ lo evaluó como poco atractivo, peligroso e inseguro. El Lugar Tal Como esl evocó percepciones de desorden físico moderado. El Lugar Mejorado se percibió como tranquilo, verde y bien conservado, y alrededor del $30 \%$ lo considero privado, atractivo y seguro.

\section{Nivel de desorden, riesgo y seguridad}

Con el fin de identificar constructos latentes en la escala de desorden físico y social, se realizo un análisis factorial de tipo exploratorio considerando las respuestas obtenidas para los tres lugares evaluados.. Se extrajeron cuatro factores: el Factor I, orden físico, se refiere a las condiciones físicas del lugar y a que tanto le gusta a la gente (alfa de Cronbach $=.97)$. El Factor II, desorden social, incluye atributos asociados al comportamiento antisocial y la degradación en lugares pobres y descuidados (Alfa de Cronbach $=.92$ ). El Factor III, sentido de comunidad, refleja buenas relaciones sociales y vida comunitaria entre los residentes (Alfa de Cronbach $=.90$ ). Finalmente, el Factor IV, se refiere a la medida en la que el lugar tiene buena visibilidad (Alfa de Cronbach $=$ $.61)$.

Considerando los factores extraídos, se realizaron una serie de Análisis de Varianza (ANOVAS) y de pruebas $\mathrm{T}$ con el fin de conocer si los tres lugares evaluados se 
percibían significativamente diferente y si existían diferencias por edad y sexo. La Tabla II muestra las medias y desviaciones estándar de los factores extraídos para cada lugar. Los resultados muestran que el Lugar Deteriorado se percibió como el lugar más desordenado física y socialmente, y en el cual 1 a visibilidad, el sentido de comunidad y la seguridad son bajos. El Lugar tal Como es se consideró un lugar físicamente desordenado y no muy atractivo, aunque no se percibió como un lugar socialmente desordenado. El Lugar Mejorado obtuvo la evaluación más favorable, ya que se percibió como un lugar física y socialmente ordenado. Pese al hecho de que la visibilidad, el sentido de comunidad y la seguridad fueron moderados para estos dos últimos lugares, el Lugar Mejorado siempre fue mejor evaluado.

Tabla II.

Puntuaciones medias y desviaciones típicas para los factores extraídos en desorden percibido y seguridad

\begin{tabular}{|c|c|c|c|c|c|}
\hline & \multirow[b]{2}{*}{$\begin{array}{c}\text { I. } \\
\text { Relacionados } \\
\text { con el lugar }{ }^{1} \\
m(s t d)\end{array}$} & \multicolumn{2}{|c|}{$\begin{array}{c}\text { DESORDEN PERCIBIDO } \\
\text { (factores extraídos) }\end{array}$} & \multirow[b]{2}{*}{$\begin{array}{c}\text { IV. } \\
\text { Buena } \\
\text { visibilidad } \\
m(s t d)\end{array}$} & \multirow[t]{2}{*}{ SEGURIDAD } \\
\hline & & $\begin{array}{c}\text { II. } \\
\text { Orden social }^{2} \\
m(s t d)\end{array}$ & $\begin{array}{c}\text { III. } \\
\text { Sentido de } \\
\text { comunidad }^{3} \\
m(s t d)\end{array}$ & & \\
\hline$\%$ varianza explicada & 49.69 & 8.49 & 4.34 & 3.90 & N/A \\
\hline Alfa de Cronbach & .97 & .92 & .90 & .61 & .83 \\
\hline Lugar Deteriorado & $2.48(0.74)$ & $6.36(1.04)$ & $3.57(1.17)$ & $4.70(1.64)$ & $2.79(0.96)$ \\
\hline Lugar Tal Cual & $4.94(1.43)$ & $4.43(1.32)$ & $5.14(1.27)$ & $6.03(1.50)$ & $4.55(1.06)$ \\
\hline Lugar Mejorado & $7.24(1.19)$ & $2.94(0.92)$ & $5.98(1.36)$ & $6.35(1.64)$ & $5.44(0.93)$ \\
\hline \multicolumn{6}{|c|}{$1=$ En absoluto; $10=$ Muchísimo } \\
\hline \multicolumn{6}{|c|}{$\begin{array}{l}1 \text { Diferencias significativas entre los tres lugares }(\mathrm{F}(2,198)=630.935, p<.001 \text {; ajuste Bonferroni). } \\
2 \text { Diferencias significativas entre los tres lugares }(F(2,228)=374.646, p<.001 \text {; ajuste Bonferroni). } \\
3 \text { Diferencias significativas entre los tres lugares (correción Greenhouse-Geisser } F(2,207)=143.502, p<.001 \text {; ajuste } \\
\text { Bonferroni). } \\
4 \text { Diferencias significativas entre el Lugar Deteriorado y los otros dos lugares, pero no entre el Lugar Tal Cual y el Lugar } \\
\text { Mejorado (correción Greenhouse-Geisser } F(2,218)=57.078, p<.001 \text {; ajuste Bonferroni). } \\
5 \text { Diferencias significativas entre los tres lugares }(F(2,224)=349.710, p<.001 \text {; ajuste Bonferroni). }\end{array}$} \\
\hline
\end{tabular}

Los participantes percibieron que los tres lugares difieren significativamente entre sí con respecto a su nivel de desorden y seguridad. El Lugar Deteriorado obtuvo la evaluación menos favorable en todos los casos y el Lugar Mejorado la mejor. Los 
resultados mostraron diferencias significativas $(p<0.001)$ entre los tres lugares para la mayoría de los factores asociados con el desorden físico y social, excepto para el Factor IV, buena visibilidad, en el que no se encontraron diferencias significativas entre el Lugar tal Como es y el Lugar Mejorado. Igualmente los tres lugares fueron percibidos como significativamente diferentes en relación con su nivel de seguridad $(p<.001)$.

Por otro lado, se encontraron pocas diferencias entre grupos. Las mujeres ( $m=5.09 ; \mathrm{std}$ $=2.21)$ tendieron a puntuar significativamente más alto que los hombres $(m=4.16 ;$ std $=1.78$ ) en los atributos asociados al primer factor. Es decir, las mujeres hicieron una evaluación más favorable respecto de que tan atractivos y cuidados estaban los tres lugares $(\mathrm{t}(101)=2.378 ; p<.05)$. Con relación al factor de seguridad también se encontraron diferencias significativas en las que los participantes de mayor edad $(m=$ 4.80 , $s t d=1.24)$ parecían sentirse más seguros que los más jóvenes $(m=3.88$, $s t d=$ $1.4),(\mathrm{F}(2,119)=4.069, p<.05)$.

De forma parecida a la escala de desorden, se llevó a cabo un análisis factorial de tipo exploratorio en la escala de percepción de riesgo, y se extrajeron cuatro factores (Tabla III). Mientras que los primeros dos factores, delitos menores y graves (alfa de Cronbach $=.92$ y .87 , respectivamente) se refieren a delitos que pueden ser perpetrados por otros, el Factor III, controlabilidad (alfa de Cronbach $=.65$ ), está más relacionado con la capacidad personal de manejar cualquier situación inesperada; y el Factor IV con la vulnerabilidad o susceptibilidad de una persona de sufrir daños o de tener problemas (alfa de Cronbach $=.75)$.

Considerando los factores extraídos, se realizaron una serie de Análisis de Varianza (ANOVAS) y de pruebas $\mathrm{T}$ con el fin de conocer si los tres lugares evaluados se 
percibían significativamente diferentes y si existían diferencias por edad y sexo. La Tabla III muestra que los participantes percibían más riesgos en el Lugar Deteriorado. Los participantes expresaron sentirse mas vulnerables y con menos control, y que la probabilidad de ocurrencia de delitos (tanto menores como mas serios) era mayor en este lugar, comparado con los otros dos lugares. Aun cuando el Lugar tal Como es y el Lugar Mejorado fueron considerados como lugares de bajo riesgo, dicha percepción era aún menor en el Lugar Mejorado.

Con respecto a las comparaciones por edad y sexo, únicamente los factores III y IV (controlabilidad y vulnerabilidad, respectivamente) mostraron diferencias por grupos. Las mujeres $(m=3.88 ; s t d=1.19)$ tendieron a puntuar significativamente más alto que los hombres $(m=3.33 ;$ std $=0.90)$ en relación con la percepción de vulnerabilidad y la posibilidad de sufrir daños $(t(118)=2.864 ; p<.01)$.

Tabla III

Puntuaciones medias y desviaciones típicas para los factores extraídos en los que se encontraron diferencias significativas

\begin{tabular}{|c|c|c|c|c|}
\hline & \multicolumn{4}{|c|}{$\begin{array}{l}\text { PELIGRO PERCIBIDO } \\
\text { (factores extraídos) }\end{array}$} \\
\hline & $\begin{array}{c}\text { I. } \\
\text { Delitos menores }{ }^{1}\end{array}$ & $\begin{array}{l}\text { II. } \\
\text { Delitos graves }^{2}\end{array}$ & $\begin{array}{c}\text { III. } \\
\text { Control }^{3}\end{array}$ & $\begin{array}{c}\text { IV. } \\
\text { Vulnerabilidad }^{4}\end{array}$ \\
\hline$\%$ varianza explicada & 44.28 & 9.69 & 7.42 & 5.25 \\
\hline Alfa de Cronbach & .92 & .87 & .65 & .75 \\
\hline Lugar Deteriorado & $5.31(.69)$ & $4.45(1.00)$ & $3.50(1.08)$ & $4.57(1.01)$ \\
\hline Lugar Tal Cual & $3.74(.97)$ & $3.16(1.02)$ & $4.49(0.96)$ & $3.45(0.92)$ \\
\hline Lugar Mejorado & $2.59(.74)$ & $2.69(1.04)$ & $5.01(0.94)$ & $2.06(0.96)$ \\
\hline \multicolumn{5}{|l|}{ 1= En absoluto; $7=$ Muchísimo } \\
\hline \multicolumn{5}{|c|}{$\begin{array}{l}1 \text { Diferencias significativas entre los tres lugares }(F(2,218)=475.797 ; p<.001 \text { ajuste Bonferroni). } \\
2 \text { Diferencias significativas entre los tres lugares (correción Greenhouse-Geisser } F(2,200)=135.781 ; p<.001 \text { ajuste } \\
\text { Bonferroni). } \\
3 \text { Diferencias significativas entre los tres lugares }(\mathrm{F}(2,222)=107.742, p<.001 \text { ajuste Bonferroni). } \\
4 \text { Diferencias significativas entre los tres lugares (correción Greenhouse-Geisser } F(2,211)=170.194 ; p<.001 \text { ajuste } \\
\text { Bonferroni). }\end{array}$} \\
\hline
\end{tabular}


Contrario a lo esperado, los resultados muestran que cuanto mayor es una persona, menos vulnerable $(F(2,118)=5.388 ; p<.01)$ y con más control $(F(2,116)=5.766 ; p<$ .01) se siente. Es decir, los participantes entre 26 y 30 años se sentían menos vulnerables $(m=3.89$, std $=1.13)$ y con más control $(m=4.87$, std $=0.92)$ que aquellos entre 18 y 21 ( $m=4.72, s t d=1.08 ; \mathrm{y}, m=4.00, s t d=.1 .15$, respectivamente $)$. No se encontró ninguna otra diferencia significativa.

Resumiendo, los resultados demostraron que los participantes percibieron los tres lugares como significativamente diferentes en función de su nivel de desorden, riesgo y seguridad. El Lugar Deteriorado obtuvo en todos los casos la evaluación menos favorable y el Lugar Mejorado la más favorable.

\section{Discusión}

La Teoría de las Ventanas Rotas y las incivilidades sostienen que el desorden físico y social evidente nos señala que la gente es desinteresada y poco cuidadosa, lo que a su vez puede considerarse como una advertencia de la posibilidad de ocurrencia de ciertos peligros y crímenes graves. Según esta perspectiva, a mayor incivilidad y desorden percibidos, mayor inseguridad se podrá percibir. El estudio que aquí se ha presentado investiga si dicha premisa se mantiene cuando a las personas se les permite expresar libremente sus ideas acerca de un lugar, antes de evaluar mediante un cuestionario que tan desordenado o riesgo le parece dicho lugar.

En primer lugar, los resultados mostraron que la percepción de lugar puede o no incluir incivilidades sociales y juicios de seguridad. Cuando se permitió a los participantes expresar libremente sus ideas sobre los lugares evaluados en este estudio, 
principalmente mencionaron atributos asociados al desorden físico $y$ al nivel económico. Solo una minoría incluyó factores sociales y afectivos tales como como el atractivo del lugar y la amabilidad de los residentes. Las categorías que se mencionaron con más frecuencia fueron las relacionadas con incivilidades físicas, el mantenimiento y el nivel económico. Sin embargo, y contrario a lo planteado por la Teoría de las Ventanas Rotas, el desorden percibido no evocó sentimientos de inseguridad en los participantes, tal como lo demuestra la evaluación del Lugar Deteriorado, en el que solo una cuarta parte de los participantes hicieron referencia a la inseguridad del lugar.

Por otra parte, cuando se pidió explícitamente a los participantes que evaluaran cuán peligroso o inseguro era un lugar, los resultados confirman los hallazgos de otros investigadores en el campo (Cozens, Hillier y Prescott, 2001; Doran y Lees, 2005; Farrall et al., 2007; Hale, 1996; Kuo y Sullivan, 2001; Painter, 1996; Perkins y Taylor, 1996; Van Beek Gert, 2005). Es decir, cuanto más se perciba un lugar como desordenado, más inseguro y en riesgo uno se podrá sentir. Sin embargo, existe una diferencia importante entre las conclusiones de investigaciones previas y este estudio. Los resultados muestran que el lugar percibido como más inseguro y riesgoso era al mismo tiempo el más desordenado física y socialmente. Los otros dos lugares se evaluaron como socialmente ordenados, más seguros y con bajo riesgo; aun cuando el Lugar tal Como es se percibió como físicamente desordenado. Es decir, el nivel de desorden social diferenciaba a los lugares seguros de los inseguros. Esto nos indica que la percepción de seguridad de un lugar no procede del ambiente físico en sí mismo sino de las inferencias de tipo social que se hacen de un lugar, son las personas y su comportamiento lo que más importa. 
Los resultados también sugieren que es más probable encontrar diferencias por edad y sexo en relación con el mantenimiento del lugar, la vulnerabilidad y el control percibidos. Las mujeres y los participantes más jóvenes tienden a sentirse más vulnerables y con menos control que los hombres y las personas mayores. Igualmente, las mujeres mostraron una percepción más favorable de los lugares, principalmente en lo que respecta al mantenimiento y grado de atracción de los mismos.

La segunda conclusión tiene que ver con los métodos empleados. Contrario a otras investigaciones en el campo, el estudio aquí presentado emplea tres metodologías diferentes para medir el desorden, el riesgo y la seguridad. Esto facilito un mejor entendimiento de los conceptos evaluados. Anteriormente, se han empleado fotografías para provocar reacciones en los participantes, pero solía tratarse de fotografías de una sola toma, con poca o ninguna información sobre el vecindario. Con base en estudios cualitativos previos (realizados por los autores de este articulo) y en la literatura existente, en esta investigación se crearon y emplearon fotografías panorámicas de mayor riqueza visual.. Mientras que la mayoría de los estudios en este campo han evaluado únicamente el efecto que las áreas deterioradas tienen en la percepción de miedo e inseguridad, el presente estudio también investigó el efecto que tienen tanto los lugares desordenados como aquellos ambientalmente mejorados, con el fin de corroborar los supuestos .conceptuales en el campo del miedo al crimen..

Aun cuando las modificaciones efectuadas a la fotografía del lugar original no solo incluyeron algunos de los atributos que según la literatura contribuyen significativamente a la percepción de seguridad de la gente, sino también otros aspectos tales como la vegetación, el tipo de carreteras y el número de autos, todavía queda por 
determinar si fue la presencia de los primeros atributos o más bien el efecto combinado de todos ellos, lo que influyo la percepción de lugar. Es por tanto necesario investigar más a fondo cuántos y cuáles atributos son suficientes para evocar percepciones de inseguridad.

Los resultados de este estudio también evidenciaron varias cuestiones metodológicas importantes. El análisis cualitativo sugiere que a pesar del nivel de desorden físico percibido por la mayoría de los participantes, no se percibieron desorden social o sentimientos de inseguridad. Al contrario, las respuestas a los cuestionarios muestran que los lugares físicamente desordenados se asocian con incivilidades sociales y falta de seguridad. Esto confirma los hallazgos de otras investigaciones (Farrall et al., 1997; Fattah, 1993 0). Los resultados de este tipo deben interpretarse con precaución ya que pueden resultar engañosos, pues no toman en cuenta las primeras impresiones sino las respuestas a preguntas cerradas sobre el desorden y la seguridad. Lo que parece evidente, es que según los métodos y las técnicas empleados para investigar este tema se pueden obtener conclusiones distintas. La estimación del nivel de seguridad puede o no ser parte de la evaluación personal del lugar, pero también puede ser el resultado de las demandas de la investigación.. Es necesario combinar técnicas cualitativas y cuantitativas, así como desarrollar nuevas e innovadoras formas de medir la percepción de seguridad, de riesgo y otros conceptos relacionados. Por otra parte, es necesario definir más claramente los conceptos importantes, y medir de manera creativa las reacciones al crimen y a la seguridad. Asimismo, se deberían investigar los contextos espacio-temporales y sociales del fenómeno en cuestión. 


\section{Conclusiones}

Durante más de 20 años la Teoría de las Ventanas Rotas y otros desarrollos conceptuales han guiado la investigación científica, las políticas sociales y el diseño urbano. Sin embargo, el éxito aparente de este enfoque parece tener más relación con la relativa facilidad con que se pueden instrumentar las políticas y medidas de diseño que con su efectividad para reducir la percepción de inseguridad y el crimen.

Los resultados de este estudio sugieren que los indicadores de peligro no son evidentes. Por lo tanto, la creencia de que las características visibles de deterioro y degradación ambiental son muestras de incivilidad social y de peligro es incompleta, ya que tales características por si mismas no presentan peligros. Más bien, la gente interpreta el ambiente en función de quiénes son, de dónde vienen y por qué están ahí (Goffman, 1971; Warr, 1990). La implicación de estos resultados es que no solo distintas personas realizan distintas evaluaciones acerca del desorden y la seguridad, sino que la modificación de las características físicas de los lugares no es suficiente para reducir el crimen y los sentimientos de inseguridad. Mediante la combinación de metodologías cuantitativas y cualitativas para investigar los puntos de vista del público y sus percepciones de desorden, peligro y seguridad, la investigación futura debe también centrarse en la interacción entre factores psicológicos, ambientales y socioculturales, estableciendo el contexto especifico en el que ocurre dicha interacción. 


\section{Referencias}

Brantingham, P. \& Brantingham, P. (1995). Criminality of place. Crime generators and crime attractors. harrsid5981147 European Journal on Criminal Policy and research, 3, 5-26.

Brown, B. B. Perkins, D. D. \& Brown, G. (2004). Incivilities, place attachment and crime: Block and individual effects. Journal of Environmental Psychology, 24, 359371.

Brown, B. B. \& Perkins, D. D. (2001). Neighbourhood revitalization and disorder: An intervention evaluation. Executive summary of final report to The National Institute of Justice. Salt Lake City: University of Utah. Recuperado de: http://www.people.vanderbilt.edu/ douglas.d.perkins/NIJsum.final.htm

Brunson, L., Kuo, F. E. \& Sullivan, W. C. (2001). Resident appropriation of defensible space in public housing: Implications for Safety and Community. Environment and Behavior, 33 nsrsid5981147(5), 626-652.

Brunson, L., Kuo, F. E. \& Sullivan, W. C. (2001). Resident appropriation of defensible space in public housing: Implications for Safety and Community. Environment and Behavior, 33 (5), 626-652.

Cozens, P., Hillier, D. \& Prescott, G. (2001). Crime and the design of residential property: Exploring the theoretical background (Part 2). Property management, 19 (2), 136-164. ng1033

Doran, B. J. \& Lees, B. G. (2005). Investigating the spatiotemporal links between disorder, crime, and the fear of crime. The Professional Geographer, 57 (1), 1-12.

Farrall, S., Bannister, J., Ditton, J. \& Gilchrist, E. (1997). Questioning the measurement of the fear of crime. Findings from a Major Methodological Study. British Journal of Criminology, 37 (4), 658-679. 
Farrall, S., Gray, E. \& Jackson, J. (2007). 'Theorising the Fear of Crime: The Cultural and Social Significance of Insecurities about Crime', Experience and Expression in the Fear of Crime: Working Paper \#5. Disponible en SSRN: http://ssrn.com/abstract $=1012393$.

Fattah, E. A. (1993). Research On Fe ar of Crime: Some Common Conceptual And Measurement Problems. En W. Bilsky, C. Pfeiffer \& P. Wetzels (Eds.), Fear of Crime and Criminal Victimisation. Stuttgart: Ferdinand Enke Verlag.

Ferraro, K. F. \& LaGrange, R. (1987). The measurement of fear of crime, Sociological Inquiry, 57 (1), 70-101.

Franzini, L., O’Brien, M., Murray, S. \& O’Campo, P. (2008). Perceptions of disorder: Contributions of neighbourhood characteristics to subjective perceptions of disorder. Journal of Environm ental Psychology, 28, 83-93.

Friederichs, J. \& Blasius, J. (2003). Social norms in distressed neighbourhoods: Testing the Wilson Hypothesis. Housing Studies, 18 (6), 807-826.

Garcia, R. M., Taylor, R. B. \& Brian, A. L. (2007). Impacts of violent crime and neighbourhood structure on trusting your neighbours. Justice Quarterly, 24 (4), 679704.

Goffman, E. (1971). Relations in public: Mic rostudies of the public order. Great Britain: Cox and Wyman Ltd (Penguin Books, Ltd.).

Hale, C. (1996). Fear of Crime: A review of the literature. International Review of Victimology, 4, 79-150.

Jackson, J. P. (2004a). Experience and expression: Social and cultural significance in the fear of crime. British Journal of Criminology, 44, 946-966. 
Jackson, J. P. (2004b). An Analysis of construct and debate: The fear of crime. In H. Albrecht, T. Serassis, \& H. Kania (Eds.), Images of crime II. Freiburg: Edition Luscrim (Max Planck Institute).

Kuo, F. E., Bacaicoa, M. \& Sullivan, W. C. (1998). Transforming Inner-City landscapes. Trees, sense of safety and prefere nce. Environment and Behavior. 30 (1), 28-59.

Kuo, F. E. \& Sullivan, W. C. (2001). Environment and crime in the Inner city: Does vegetation reduce crime? Environment and Behavior, 33 (3), 343-367.

Markowitz, F. E., Bellair, P. E., Liska, A. \& Liu, J. (2001). Extending Social Disorganisation Theory: Modeling the relationships between cohesion, disorder and fear. Criminology, 39 (2), 293-319.

Miceli, R., Roccato, M. \& Rosato, R. (2004). Fear of crime in Italy. Environment and behaviour, 36 (6), 776-789.

Miles, M. B. \& Huberman, A. M. (1994). Qualitative Data Analysis: An Expanded Sources Book. Thousands Oaks, CA: SAGE Publication.

Painter, K. (1996). The influence of street lighting improvements on crime, fear and pedestrian street use, after dark. Landscape and Urban Planning, 35, 193-201.

Perkins, D. D. \& Taylor, R. B. (1996). Ecological assessments of community disorder: Their relationship to fear of crime and theoretical implications. American Journal of Community Psychology, 24, 63-107.

Ross, C. E., Reynolds, J. R. \& Geis, K. J. (2000). The contingent meaning of neighbourhood stability for resident's psychological well-being. American Sociological Review, 65 (4), 581-597. 
Schweitzer, J. H., Woo-Kim, J. \& Mackin J. R. (1999). The impact of the built environment on crime and fear of crime in urban neighbourhoods. Journal of urban technology, 6 (3), 59-73.

Semmens, N. (2004). The intangible costs of crime: Why the fear of crime causes us a problem. Paper to the second ESRC seminar on "Crime, insecurity and well-being: an economic approach". Shefield, 18 May, 2004. Paper obtained from the author.

Skogan, W. G. (1990). Disorder and decline: Crime and the spiral of decay in American neighbourhoods. Los Angeles: University of California Press.

Taylor, R. B. (2001a). Breaking away from broken windows. Boulder, CO: Westview Press.

Taylor, R. B. (2001b). Crime prevention through environmental design: Yes, no, maybe, unknowable, and all of the above. En R. Bechtel \& A. Churchman (Eds.): Handbook of Environmental Psychology (pp. 413-425). USA: John Wiley and Sons, Inc.

Taylor, R. B. (1996). Neighbourhood responses to disorder and local attachments: The systemic model of attachment, social disorganization, and neighbourhood use value. Sociologic al Forum, 11 (1), 41-74.

Taylor, R. B. (1987). Toward an environmental psychology of disorder: delinquency, crime, and fear of crime. En D. Stokols \& I. Altman (Eds.), Handbook of Environmental Psychology (pp. 951-986). Nueva York: John-Wiley \& Sons. Van Beek Gert (2005). Fear of crime: Feeling (un)safe and (in)secure in the risk society. Recuperado de http://statbel.fgov.be/studies/ac084_en.pdf .

Walkalate, S. (1998). Crime and Community: Fear or Trust? The British Journal of Sociology, 49 (4), 550-569. 
Desorden percibido y la influencia del método

Warr, M. (1990). Dangerous situations: Social context and fear of victimization. Social forces, 68 (3), 891-907.

Weber, R. P. (1990). Basic Content Analysis. Newbury Park, CA: SAGE Pub lications.

Wilson, J. Q. (1975). Thinking about Crime. Nueva York: Basic Books.

Wilson, J. Q. \& Kelling, G. L. (1982). Broken windows. Atlantic Monthly, 211, 29-38 